Herz 2012 · 37:121-122

DOI 10.1007/s00059-012-3598-9

Online publiziert: 2. März 2012

(c) Urban \& Vogel 2012

E. Hoffmann ${ }^{1}$ - B. Maisch ${ }^{2}$

${ }^{1}$ Klinikum München-Bogenhausen, München

${ }^{2}$ Klinik für Innere Medizin, Schwerpunkt Kardiologie, Universitätsklinikum

Gießen und Marburg GmbH, Marburg

\title{
Interventionelle kardiovaskuläre Therapie
}

\section{Aktueller Stand 2012}

Die interventionelle kardiovaskuläre Therapie hat in den vergangenen Jahren eine rasante Entwicklung erfahren. Neue Technologien haben sowohl in der interventionellen Kardiologie als auch in der interventionellen Elektrophysiologie unter Einschluss der Device-Therapie Einzug gehalten. Das Schwerpunktthema der aktuellen Ausgabe der ReviewZeitschrift Herz zur 78. Jahrestagung der Deutschen Gesellschaft für Kardiologie in Mannheim, zu der dieses Schwerpunktheft erscheint, widmet sich ganz bewusst der interventionellen kardiovaskulären Therapie.

Kardiologen in der niedergelassenen Praxis brauchen ebenso wie in den Kliniken angesichts der Vielfalt der Therapiemöglichkeiten und Technologien eine aktuelle Standortbestimmung, um die jeweils individuell optimale Empfehlung an die Patienten weiterzugeben. Wie so oft haben die Berichte über neue Technologien ein Übermaß an Euphorie hervorgerufen. Denken wir nur an die Restenoserate von $0 \%$ in der RAVEL-Studie unter Einsatz der ersten „Drug-eluting“Stents. Manch eine als vielversprechend angekündigte Methode musste doch herbe Rückschläge in Kauf nehmen. Einige Verfahren sind mittlerweile Geschichte und wurden von anderen Entwicklungen überholt wie z. B. die Brachytherapie der In-Stent-Restenose. Neue Verfahren sollten sich durch harte Daten, am besten durch doppelt blind randomisierte Studien und die Bewährung im Alltag, zunächst beweisen, bevor ihr Einsatz in der
Routine empfohlen werden kann. Die zusätzlichen Kosten sollten durch einen adäquaten Nutzen in die richtige Relation gebracht werden.

Die Übersichtsarbeiten dieses Hefts, die sich alle an der aktuellen Studienlage orientieren, geben eine komprimierte Übersicht zu diesen Themen und versuchen Unsicherheiten auszuräumen.

Die „Drug-eluting“-Stents sind seit ihrer Einführung unverzichtbarer Bestandteil der Behandlung der koronaren Herzerkrankung. Stents der neuesten Generation müssen sich an ihren Vorgängern messen lassen wie der „Drug-eluting"-Ballon, so Rittger und Mitarbeiter in ihrem Beitrag, in dem sie auch zur noch immer kontrovers diskutierten Frage des Einsatzes von Stents bei koronarer Mehrgefäßerkrankung versus Bypass-Operation und zur Frage der Stent-Implantation in einen ungeschützten Hauptstamm Stellung nehmen.

Der medikamentösen Begleittherapie, hier der optimalen antithrombozytären Therapie, widmen sich Braun und Mitarbeiter. Die bisherigen Grenzen bei der Behandlung mit Clopidogrel haben die Entwicklung der neuen ADP-Rezeptor-Antagonisten Prasugrel, Ticagrelor und Cangrelor stimuliert. So profitieren Patienten mit akutem Koronarsyndrom (ACS) in der Regel von der Gabe von Ticagrelor oder Prasugrel anstelle von Clopidogrel, wobei ein direkter Vergleich der beiden neuen Substanzen noch aussteht. Da nicht alle ACS-Patienten gleichermaßen von den neuen Substanzen profitie- ren und Patienten im höheren Alter, bei Kachexie, nach Schlaganfall oder TIA in der Vorgeschichte durch eine erhöhte Blutungsneigung gefährdet sind, sollte bei ihnen auf die Gabe von Prasugrel und Ticagrelor gegenwärtig verzichtet werden.

In der Device-Therapie galten konventionelle Herzschrittmacher und implantierbare Cardioverter-Defibrillatoren (ICD) lange Zeit als Kontraindikation für die Magnetresonanztomographie (MRT), die zunehmend in vielen medizinischen Bereichen unverzichtbar wurde. So stellt die Entwicklung von MRT-kompatiblen Schrittmachern eine wichtige Innovation dar, die zukünftig zu einem neuen Standard der Geräte werden dürfte, wie Dorenkamp et al. in ihrem Beitrag zeigen. Die Risiken, die der Hintergrund der bisherigen Kontraindikationen waren, wurden bislang überschätzt. Dies führte bereits zur Anpassung der Empfehlungen der europäischen Gesellschaft für Kardiologie. Ebenso werden praktische Empfehlungen gegeben, was der betreuende Kardiologe bei einer MRT-Untersuchung bei Patienten mit Device-Implantaten beachten muss und wie ggf. mit nicht-MRTkompatiblen Sonden bei zukünftigen Wechseln auf die potenziell MRT-tauglichen Aggregate zu verfahren ist.

Die MRT spielt auch im Beitrag von Mahnkopf et al. zur Ablationstherapie mittels Kardio-MRT eine zentrale Rolle. Für die Durchleuchtung mit Röntgenstrahlen in der interventionellen Elektrophysiologie gibt es derzeit noch keinen Ersatz. Einige Gruppen arbeiten mit Hoch- 
druck an der strahlenfreien Ablation. Dreidimensionale Mapping-Systeme sind bei komplexen linksseitigen Prozeduren heute Standard. Sie haben die Untersuchungs- und Röntgenzeiten in den meisten Zentren nicht entscheidend reduzieren können. Die bisherige Pionierarbeit in der Entwicklung der MRT-fähigen Ablation gestattet Aussagen zum Ausmaß der kardialen Fibrose und kann mit Hilfe der postinterventionellen Visualisierung von induzierten Läsionen den Effekt der Ablation dokumentieren.

Die elektrophysiologisch geführte Diskonnektion der Pulmonalvenen (PV) ist in der Behandlung des Vorhofflimmerns (VHF) heute ein Eckpfeiler einer erfolgreichen Ablation, besonders beim paroxysmalen VHF. Hier kommt heute neben der Radiofrequenz- (RF) vor allem die Kryoenergie zum Einsatz. Schmidt und Mitarbeiter diskutieren u. a. die Vor- und Nachteile der Kryoablation im Vergleich zur Radiofrequenzablation.

Neben den innovativen Techniken sind in der Rhythmologie auch neue medikamentöse Therapieoptionen von großem Interesse. Zur Schlaganfallprophylaxe wurden mehrere neue Antikoagulanzien als Alternative zu Vitamin-KAntagonisten entwickelt. Kaya et al. gehen auf die wesentlichen Studiendaten von Dabigatran, Apixaban und Rivaroxaban ein, die ihren Stellenwert bei Patienten mit deutlich erhöhtem Risikoprofil und neu einzuleitender oraler Antikoagulation haben. Eine adäquat eingestellte orale Antikoagulation mit einem Vitamin-K-Antagonisten sollte aber nach aktuellem Kenntnisstand fortgeführt werden. Hinsichtlich der Rhythmuskontrolle von VHF werden mit Vernakalant und Dronedaron zwei neue Substanzen diskutiert. Nach den enttäuschenden Daten des Einsatzes von Dronedaron bei Patienten mit permanentem VHF in der PALLASStudie bleibt nur noch die Indikation für die Rezidivpropyhlaxe und die Frequenzkontrolle bei klinisch stabilen Patienten mit nichtpermanentem VHF und Herzinsuffizienz bis zum Stadium NYHA II bestehen. Vernakalant kann zur schnellen intravenösen pharmakologischen Sinusrhythmuskonversion bei hämodynamisch stabilen Patienten mit struktureller Herzerkrankung eingesetzt werden.
Wir wünschen unseren Lesern viel Freude bei der Lektüre dieser Ausgabe anlässlich der 78. Jahrestagung der Deutschen Gesellschaft für Kardiologie.

Ihre

Ellen Hoffmann

Bernhard Maisch

\section{Korrespondenzadresse}

Prof. Dr. E. Hoffmann

Klinikum München-Bogenhausen

Englschalkinger Str. 77, 81925 München

ellen.hoffmann@klinikum-muenchen.de 